\title{
The Logic of Self-Organized Criticality
}

\section{Kamil I. Bakhtiyarov}

Russian State Agrarian University, Moscow Timiryazev Agricultural Academy, Russia

e-mail: kamil.bakhtiyarov@gmail.com

\begin{abstract}
:
A consideration of non-classical logic in terms of classical one allows us to show a role of designated truth values. In this way we show that our version of non-classical many-valued logic can be based on the structure of genetic code. Keywords: many-valued logic, non-classical logic, designated truth values.
\end{abstract}

We are suckers and will gravitate toward those variables that are unstable but that appear stable.

Talib N. The Black Swan.

\section{Principles of Universal Language}

The Great Leibniz considered his main prime target to improve discovery art as such. For that he set up a problem to create the so-called universal characteristic. However, setting a problem and solving it are two absolutely different things. Ironically, in the article New Method for Maximums and Minimums... he focused on its implementation instead of considering the method in terms of metasymbols. I continue his work in the present paper and propose to use the signs of extremums as symbols of universal language.

Mathematics has the exact classification of extremums: maximums and minimums which are subdivided into smooth (derivative $y^{\prime}=0$ ) and sharp (derivative $y^{\prime}$ does not exist):

1.0. Maximums.

1.1. Sharp maximums.

1.2. Smooth maximums.

2.0. Minimums.

2.1. Sharp minimums.

2.2. Smooth minimums.

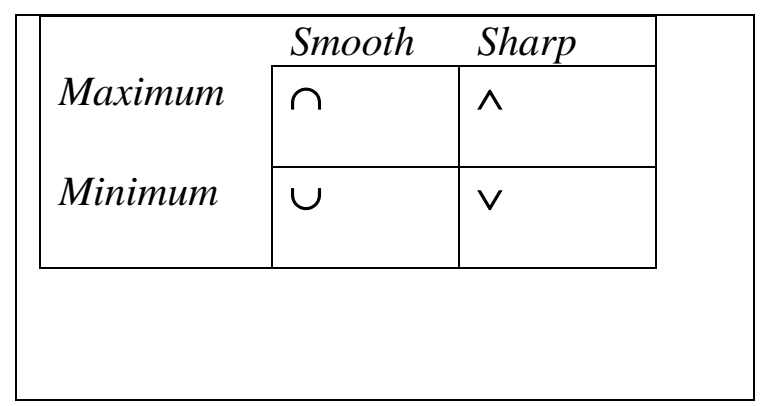

Extremums are symbols of universal language. Complementary pairs fit each other as parts and ISSN 2299-0518 
used also for letters of a genetic code [4]. Maximums $\cap, \wedge$ are used to be designated by the letters $\mathbf{n}$ and $\mathbf{A}$, and minimums $\cup, \vee$ by the letters $\mathbf{u}$ and $\mathbf{V}$.

All two-letter words are formed by application of "letters" to themselves, providing a quantum leap to the highest level. Elements of microlevel take the meaning only in a macrolevel context. In pairs $(x, y)$ a prefix $x=$ MICROLEVEL (name), and a radical $y=$ MACROLEVEL (surname). These elements of microlevel form macrolevel BLOCKS. Capital letters of radical dominants have a priority, and small letters of non-dominant radicals provide the priority to any letters of prefixes. The designation is provided by vowels and anti-designation by consonants.

Thomas Kuhn in his book The Structure of Scientific Revolutions emphasized as follows: "A decision between alternate ways of practicing science is called for, and in the circumstances that decision must be based less on past achievement than on future promise". He focused attention on the "normal" science (with a constant paradigm) that has not allowed it to describe a structure of scientific revolutions (with change of paradigms). Only after considering the logic structure of dominants and not dominants, it is possible to formulate structural principles of scientific revolutions.

The complete character of DOMINANTS is provided by submission to capital vowel or concordant of the radical and the vague character of non-dominants (with small letters of the radical) by the change of submission to vowel and concordant of the prefix. The mechanism of revolution and paradigm change can be learnt in this way. A small change in transitional demiblocks prepares the great change between blocks. The majority does not perceive a small change that generates errors of the 1st and 2nd kind. In hindsight, errors are perceived as prediction and afteraction phantoms respectively.

The living logic with the Matrix of Genesis allows defining the features matching the plan of the Nature. Creation of a block matrix (by means of the left tensor square) allowed revealing macrolevel and circular arrangement of charts. The genetic code is an information code. And in science philosophy there is no other way to follow the Nature except to follow genetic codes.

For a sample of letters of universal language, the author took letters of extremum signs. As a prototype for a symbol of a maximum there was the letter $\mathbf{A}$ (adenine), and the letter $\mathbf{u}$ (uracil) was the symbol of weak minimum. For another complementary pair, the similar turned signs were taken: a letter $\mathbf{V}$ (cytosin) as a minimum sign, and a letter $\mathbf{n}$ (guanine) as a sign of a weak maximum. The initial matrix is made up of the complementary pairs of consonants $(\mathbf{n}, \mathbf{V})$ and vowels $(\mathbf{A}, \mathbf{u})$. Accordants provide stability of the concaved (caved in under the power) consonants $* \mathbf{V}$, and discordants provide variability of convex vowels (arising dissidents) *A.

The consideration of non-classical logic in terms of classical logic allows us to show clearly the reduction role for obtaining the designated truth values. The logic reduction of non-classical multi-valuedness to classical binarity of the designated and anti-designated truth values forms overlaps from right to left and from left to right, forming a hysteresis loop arranging the circular order.

The model offered allows tracking visually the large-block construction of the logic matrix, and then the arrangement of separate cells which are filled according to the principle of similarity. A position record needs only 4 letters at the highest levels. The positioning principle is inherent for analytic approach and for humanities it offers not less advantages, than for arithmetics.

The asymptotic correspondence of classical and non-classical theories is a manifestation of the principle of compliance which establishes the connection between these representations. The complementarity of levels generates fractality (self-similarity) of the main table structure. The offered genetic method to solve the problem of transition from one paradigm to another is the message which provides the clue to cognitive process that will help fill the gap between natural intelligence and artificial intelligence. 


\section{The Logic of Self-organized Criticality: Designation and Anti-designation}

Elements form the four symbols of metascience as maximums and minimums:

$\wedge$ - a maximum, A, Adenin of genetic code and psychological type Art are designated as $A=\underline{11}$; it is a designated value;

$\checkmark$ - minimum, $\mathrm{C}$, Cytosine and psychological type Authority are designated as the reversed sign $\mathrm{V}=$ $\underline{00}$; it is an anti-designated value;

$\cap$ - weak maximum, g, the Guanine and psychological type Media are designated as the sign $\mathrm{n}=$ $\underline{01}$; it is an anti-designated value;

$\cup$ - weak minimum, Uracyl and psychological type Scientist are designated as the sign $\mathrm{u}=\underline{10}$; it is a designated value.

The alphabet of educational metasymbols solves a problem of polystructural integration of knowledge naturally through their comparison. The transition to the left tensor square allowed condensing constellation into a monolithic block [3]. The genetic table has 4 blocks of the designated and anti-designated pairs of metasymbols which are based on the universal language:

\begin{tabular}{|c|c|c|c|c|c|c|c|c|c|c|c|c|c|}
\hline $\mathrm{nn}$ & An & nA & $\overline{\mathbf{A A}}$ & \multirow{4}{*}{$\mathrm{z}=$} & $\begin{array}{lll}0 & 01 & 01\end{array}$ & 1101 & 0111 & 1111 & \multirow{4}{*}{$\mathrm{s}=$} & 2 & 3 & 3 & 4 \\
\hline $\mathrm{Vn}$ & un & $\overline{\text { VA }}$ & $\mathbf{u A}$ & & 0001 & 1001 & $00 \underline{11}$ & 1011 & & 1 & 2 & 2 & 3 \\
\hline $\mathrm{nV}$ & AV & $\mathrm{nu}$ & $\mathbf{A u}$ & & $01 \underline{00}$ & 1100 & $\underline{0} 1 \overline{10}$ & $11 \overline{10}$ & & 1 & 2 & 2 & 3 \\
\hline VV & $\mathrm{uV}$ & $\mathrm{Vu}$ & $\mathbf{U u}$ & & $00 \underline{00}$ & $10 \underline{00}$ & $\underline{0} 010$ & 1010 & & 0 & 1 & 1 & 2 \\
\hline
\end{tabular}

Everything is predetermined by dominants. Sequences are estimated through consequences:

if it is consistent, then it is dominant - the basis is considered,

if it is inconsistent, then it is non-dominant - the beginning is considered.

The macrolevel dominants have the priority (for not dominants - any letters of microlevel do) [3], [4]. For dominant values there is a reduction in roots: $\mathrm{A}=\underline{11}, 00 \underline{11}$ designated and $\mathrm{V}=\underline{00}$, 1100 the anti-designated values; for non-dominant values there is a reduction in the first figure: $\mathrm{u}=$ $\underline{10}, \underline{1010}, \underline{1001}$ designated and $\mathrm{n}=\underline{0} 1, \underline{0} 110, \underline{0} 101$ anti-designated values [2].

Instead of considering true values (z) usually only the total number of units (s) is counted. So, we have incomplete information, without order. When $s$ is more than 2 we receive designated values $\mathrm{z}$, and when $\mathrm{s}$ is less than 2 we have anti-designated values $\mathrm{z}$. Transitional value $\mathrm{s}=2$ is not decisive, because it is split into designated/anti-designated values (sat./unsat.). The transition from anti-designated $11 \underline{00}$ to the next designated value 1001 is "awakening" like shift of subthreshhold values to the overthreshhold values. Vice versa the transition from the designated value 0011 to the anti-designated next value $\underline{0} 110$ is "lulling". So, we have overthreshold values above and subthreshold values below. Between them thre is a critical zone that is characterized by ability to switch, turn quickly, as avalanche. None was familiar aith its delicate theory.

Thus, the classification of the designated and anti-designated pairs for the first time was received through genetics. Metasymbols generated new result in logic [4]. The probability theory usually uses the famous Pascal's triangle (at the left), that generates after splitting (in the center) a genetic triangle (on the right) by doubling numbers to the right of a vertical.

$\begin{array}{lll}1 \mid 1 & 1 \mid 1 & 1 \mid 2 \\ 121 & 11 \mid 11 & 11 \mid 22 \\ 13 \mid 31 & 13 \mid 31 & 13 \mid 62 \\ 1464 & 143 \mid 341 & 143 \mid 682\end{array}$


Let us count the total number of "fours" of logic codons: 8 indivisible left $+8 \times 2$ doubled right equal to 24 codons. In real genetics it provides 20 amino acids + STOP command. The total is 21 (minus 3 repeated ones). We have non-Pascal's asymmetric genetic triangle. When $\mathrm{s}=2$ the value is split into designated (the quantity doubles) and the antidesignated values. That is represented in asymmetric genetic triangle, but is not represented in a symmetric Pascal's triangle.

The classical model of probability theory fails because of inability to estimate financial risks. It is necessary to refuse from classical model in favor of fractal accident - spreading from a two-level kernel. In a dyad (bases and beginnings) everything is defined by dominants which are the highest teleologic principle of designation and anti-designation. Genetic approach allows finding logic structure on genetic principles - fundamental principles which are applied by the Nature. Rapprochement of economy and genetics unifies the science, explaining all macroscopical phenomena during the periods of crises.

Dealing with animated objects, we use Pascal's dead triangle as an artificial limb instead of live hand. After amputating the living unknown, we are unable to manage the risk. "Our aversion to variability and desire for order, and our acting on those feelings, has had precipitate severe crises... Since Plato the western thought and gnoseology focused on True/False concepts. Although it was good long ago it is time to switch to Stable/Unstable" [5]. N. Taleb applied for rare but worldshaking crises term "Black swan". It is Taleb's Fourth Quadrant, a block of convex vowels. It allows to represent variability $* \mathbf{A}$ as a cluster equal to a cluster of stability $* \mathbf{V}$ as concaved consonants. Diagonal oppositions of the designated and anti-designated true-false pairs anti-commutate in identity on designation: $\mathrm{AV}=-\mathbf{V A}$, causing a complementarity. An anicommutativity generates a qualitative transition, the small change in transitional blocks prepares a big change between blocks of dominants. The roots of complementarity are in a stability and variability problem. The principle of compliance generates fractality (self-similarity) for the structure of the main table.

Life wins due to expanded reproduction of the most adapted accidental mutations. Other disappears from a genetic bank [6]. The mutability is carried out through equiprobable options in genetic code, while the stability - through the absence of alternatives, when various options imploded. Doubling provides the raised stability during transfer of genetic information. There is an asymmetry of two clusters - designated cluster has twice more possibilities than antidesignated cluster.

Considering non-classical logic in terms of classical one allows us to show a role of designated truth values. The model of phase transitions which is a dream of P. Bak is based on the non-classical many-valued logic based on fractal structure of genetic code and isomorphism of selforganized critical theories [1].

\section{References:}

1. Bak, P. How Nature Works: The Science of Self-Organized Criticality. New York, 1996.

2. Bakhtiyarov, K.I. The dial of the circular complementarity of the designated and anti-designated Pairs, Studia Humana, vol. 1:3/4, 2012.

3. Bakhtiyarov, K.I. Logika i psihogenetika s tochki zreniia computer science (3rd edition). Moscow, 2014 (rus).

Logic and psycho-genetic from the point of view of computer science (3 edition) (eng).

4. Bakhtiyarov, K.I. Principles of Universal Language. Moscow: URSS, 2015 (rus).

5. Taleb, H. On robustness and fragility. New York, 2010.

6. Taleb, H. Antifragile. New York, 2012. 